\title{
Cross Layer based Optimal Path Selection Reactive Routing in MANETs
}

\author{
Jinesh Kumar Singh \\ Assistant Professor, CSE \\ Sir Padampat Singhania \\ University, Udaipur, (Raj) India
}

\author{
Nitin Bhardwaj \\ Assistant Professor, CSE \\ Northern Inst. of Eng. Technical \\ Campus, Alwar, (Raj) India
}

\author{
Prasun Chakrabarti \\ Professor and Head, CSE \\ Sir Padampat Singhania \\ University, Udaipur, (Raj) India
}

\begin{abstract}
In MANETs, routing is a complex task as many factors affect the network performance. The major factors in routing are node mobility, node energy and congestion in the wireless bandwidth limited channel and battery operated nodes with dynamic topology. A new routing protocol is proposed (OPSAODV) to select an optimal path in MANET by including the node energy and congestion status in route selection process. A cross layer approach is used to address the issue of node mobility by monitoring the received node signal power (RNSP), which indicates the node movement with respect to another node. The RNSP is used to address the node mobility issue while energy and congestion metric are used in selecting optimal path between source and destination.
\end{abstract}

\section{General Terms}

Mobile Ad Hoc Networks, Routing in MANETS

\section{Keywords}

MANETs, AODV, Optimal Path Selection, OPS-AODV, RNSP.

\section{INTRODUCTION}

Wireless ad hoc network is a collection of mobile nodes, operated on battery power and equipped with a transmitter and receiver. All the nodes are connected to each other without having a central coordinator. MANET setups do not require a pre-established infrastructure or back bone network. The nodes communicate to each other using a cooperative routing process for data transfer. Each node works as host as well as a router in MANET. These features make the MANETs highly dynamic and deployable in a variety of situations including natural disasters, military operations and communication in areas where either infrastructure network is not available or inconvenient to setup and use.

Certain issues arise in MANET because of inherent limitations of the mobile nodes and dynamic nature of the setup, like node mobility. Node mobility is an advantage to the users, that services and resources can be access from anywhere while on the move. The disadvantage is that mobility cause topology change, existing routing path changes and overhead increases in searching new paths. High mobility makes the network unstable unless detection and proactive actions are used against mobility from a network point of view [1].

Another issue in MANET is congestion which can degrade performance of the network. A network congestion situation is comparable with traffic jam on road. When the incoming traffic exceeds the limit of resources (it can be the node buffer size or channel bandwidth), the packets start dropping from the queue. During congestion packets suffer from higher than normal delay, waiting in the queues somewhere in the network along the path between source and destination. The effect of this packet loss and higher delay is under utilization of complete network resources and reduced throughput. The problem of congestion is one of the reasons of packet drop in MANETs [2].

One more related issue in MANET is energy awareness in routing path selection. As the nodes are battery operated, it requires an efficient power utilization mechanism which consumes minimum possible power from nodes. If a node goes into power drain state and shutdown itself during data transfer, the connections going through it will break, which will cause packet loss and overhead of finding new path between source and destination. It will also reduce network life time if many such nodes are included in the routing paths. If a particular node is near to battery exhaust condition, routing path selection mechanism must exclude such nodes to increase the node life time as well as overall network lifetime and to reduce packet loss caused by power drained nodes. Therefore, an efficient routing protocol must be aware of the nodes battery power so that low energy nodes are not selected in routing path [3].

The energy, congestion and mobility issues are related with each other when addressing high QoS and optimal routing path in MANET. The congestion increases, results in packet drops and this cause energy loss in the transmission and reception of the dropped packets which could be save be using a congestion adaptive policy. Similarly, mobility increase, path breaks and link fails, which results in packet drop. This energy can be saved by using a link monitoring policy to detect node mobility based on received node signal power and find alternate path before the link actually breaks. Thus all these issue must be addressed together to achieve high QoS and optimal routing path in MANETs.

There are many routing protocols designed for MANET routing. All of these protocols can be categorised into three groups: proactive like DSDV [4], reactive like AODV [5] and DSR [6] and hybrid like ZRP [7]. Proactive routing protocol tries to maintain network state by exchange routing information and as a result not very scalable and suffer from heavy overhead. Reactive routing protocols search routing path only when it is needed, which increases the performance over other protocols by reducing overhead and thus make it scalable. The hybrid routing protocol combines both proactive and reactive features by defining a Zone. Inside the zone it uses proactive policy and outside the zone it uses reactive policy. Thus the performance if such protocols depends on zone size, initial path requests and network topology.

All of these routing protocols provide a path between source and destination but none of them include metric for congestion, energy and mobility, while selecting a routing 
path. Due to this reason, these protocols find the shortest path between source and destination but not the optimal path considering mobility, energy status and congestion status of intermediate nodes. This paper present a new routing protocol based on reactive policy (AODV), which includes these metrics while selecting a path by using cross layer concept.

The traditional MANET routing protocols are based on "layered design approach" which state that all layers should be as isolated as possible in terms on information sharing in between the layers. Each layer performs a specific separate operation and then complete operation is achieved by these sub operation. This results in sub optimal performance, especially in wireless scenario in which challenges are quite different form wired scenario [8].

To fully optimize wireless networks, the challenges from the physical and MAC layers and QoS demands from the applications layers have to be taken into consideration. This is known as "Cross layer Design" approach and enforces the information sharing across the protocol stack. For example the rate, coding and power at physical can be adapted to meets the requirements of the applications, given the current channel and network conditions. The information must be shared between layers to achieve highest level of adaptability [9]. Figure 1 shows the cross layer design approach.

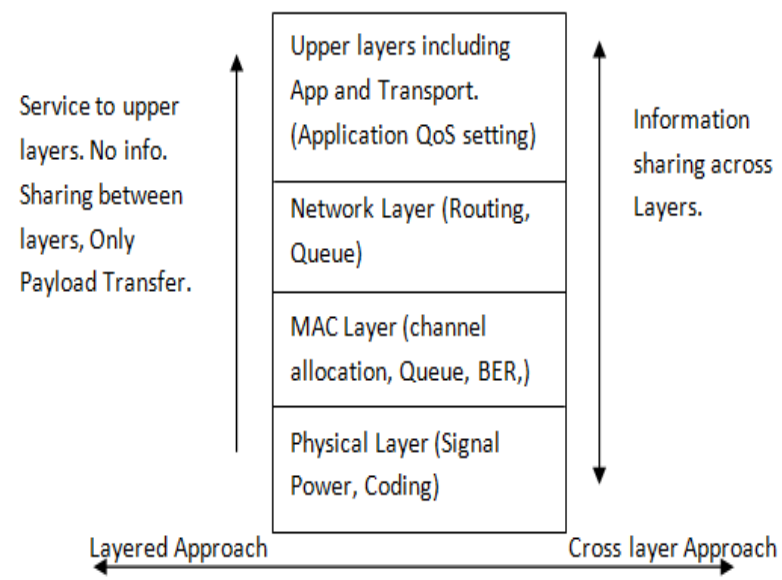

Fig 1: Cross layer Design Approach

In this paper the cross layer information used is Received Node Signal Power (RNSP), which is calculated in NS2 simulation tool at MAC layer [10]. The value of RNSP is dependent on propagation model used. The Two Ray ground reflection model is used for the proposed protocol evaluation. The Two Ray ground reflection model considers both the direct path and a ground reflection path. The Node's Received signal power calculation is shown in equation (i).

$$
\operatorname{Pr}=\frac{P t G t G r H t^{2} H r^{2}}{\mathrm{~d}^{4} L} \ldots \text { (i) }
$$

Where Pt is the transmitted signal power, Gt and Gr are the antenna gains of the transmitter and the receiver respectively. $\mathrm{Ht}$ and $\mathrm{Hr}$ is the antenna height for the transmitter and the receiver. $\mathrm{L}$ is the system loss, and $\lambda$ is the wavelength. It is common to select $\mathrm{Gt}=\mathrm{Gr}=1$ and $\mathrm{L} \geq 1$ in ns simulations.

The rest of the paper is organized as follows: Section 2 describe related work and approaches towards the issues of power, congestion and channel conditions. Section 3 briefly describes the proposed (OPS-AODV) schema to select optimal path and find alternate route, if it is needed. Section 4 explains the simulation details and results. Section 5 draws the conclusion and future scope of the work.

\section{RELATED WORK}

V. Rishiwal et al. [11] proposed QoS based power aware routing protocol (Q-PAR). The route selection metrics are energy and bandwidth. DSR routing protocol is modified to include these two requirements. In case of a link failure an alternate path is searched locally to handle the situation, which enhanced the network lifetime.

S. Singh et al. [12] proposed Minimum Battery Cost Routing (MBCR). MBCR routing protocol calculates the sum of the residual power of all nodes in a path, which is used for selecting the route between the source and destination. But the method does not consider individual node's residual power and may choose a path in which there may be mobile nodes with low power. The proposed mechanism enforces energy fairness on the network.

P.K. Suri et al. [13] proposed a bandwidth-efficient power aware routing protocol "QEPAR". The routing protocol addresses the issue of delay and bandwidth. QEPAR helps in increasing the throughput by decreasing the packet loss due to non availability of node having enough battery power to retransmit the data packet to next node. The proposed protocol is also helpful in finding out an optimal path without any loop.

T.S. Kumaran et al. [14] proposed another congestion control protocol for controlling congestion in AODV named as Early Detection Congestion and Control Routing in MANET (EDAODV), which detects congestion at the node. It calculates queue status value and thus finds the status of the congestion. Further, the non-congested predecessor and successor nodes of a congested node are used by it for initiating route finding process bi-directionally in order to find alternate non-congested path between them for sending data. It finds many alternate paths and then chooses the best path for sending data.

A. Nedumaran and V. Jeyalakshmi [15] proposed a combined energy and congestion metric based routing protocol Congestion and Energy Aware Routing Protocol (CAERP). It is based on DSR and uses variable data rates of nodes to control congestion. The protocol uses cross layer information, Received Signal Strength Indicator (RSSI) for distance estimation and queue size of node for congestion estimation. The nodes with high queue size, which are under congestion, are excluded from data rate variation. For other nodes, the data rate is changed according to the queue size and RSSI value. The results indicate reduce in congestion, energy utilization and improvement in throughput.

S. P. Terdal et al. [16] proposed energy aware and load balancing multipath routing protocol (ELB-MRP) which formulates a combined traffic and energy cost to optimize the routing mechanism by encompassing interference caused due to neighbour effect into routing decisions along with energy conservation. Contention window size and queue size are used to assess the load at a node and its one hop neighbours. Energy is also used in routing decision. Simulation results show that the performance increased with the proposed method.

\section{PROPOSED WORK 3.1 Control Variables}

The proposed protocol monitors 3 control variables as follows: 


\subsubsection{Received Node signal Power (RNSP)}

The Received node signal power is used to monitor the channel condition and node mobility to determine link stability. Each node gets RNSP value from MAC layer to network layer under cross layer design approach. If the RNSP value is going lower than the Average calculated RNSP for a particular node, than it indicates the link quality is degrading because the node is moving further. The Hello packet is used to transfer the RNSP value to the neighbour, so that each node can calculate the average RNSP (AVG_RNSP). The Hello packets are also used to maintain neighbour table with neighbour ID and its RNSP. The table is also used to find alternate routing path. A node will not forward RREQ if it is received from a node with Lower than AVG_RNSP. If the node is currently on active route then an alternate path is searched if its RNSP is lower than AVG_RNSP.

\subsubsection{Remaining node power (REM_POW)}

The Remaining node power is monitored by each node and included in RREQ packet for Route Request. Each intermediate node which forwards the RREQ towards destination adds its remaining power in RREQ. So that destination gets remaining total power of the path. The destination node waits for a predefined time to collect all the RREQs and Reply only for the highest value of the total path energy. Also a node will drop RREQ if REM_POW < EN_TH1. A node will initiate alternate path discovery if the node is currently on active route and REM_POW $<$ EN_TH2.

\subsubsection{Node queue status (QUEUE_SIZE)}

The node queue size indicates the current congestion situation for a particular node. If the QUEUE_SIZE > CO_TH1 then node will start dropping all RREQs. A node will initiate alternate path discovery if the node is currently on active route and QUEUE_SIZE > CO_TH2.

\subsection{Threshold Values}

Threshold values for the proposed OPS-AODV are defined as follows:

1. EN_TH1 is $20 \%$ of initial node power

2. EN_TH2 is $10 \%$ of initial node power

3. CO_TH1 is 80 of the total buffer capacity

4. CO_TH 2 is $90 \%$ if the total buffer capacity

5. AVG_RNSP is the average RNSP for all the neighbours of a node.

\subsection{Proposed Routing Algorithm}

1. For each RREQ packet arrived:

If $\mathrm{RNSP} \geq \mathrm{AVG}$ _RNSP \& REM_POW $\geq \mathrm{EN}$-TH1 $\&$ QUEUE SIZE $\leq$ CO_TH1

Process the RREQ with normal AODV Flow (discard if duplicate else forward) and exit.

Else

Drop RREQ and exit.

2. For each data packet arrived:

If $\mathrm{RNSP} \geq \mathrm{AVG}$ _RNSP \& REM_POW $\geq \mathrm{EN}$ _TH 2 $\&$ QUEUE_SIZE $\leq$ CO_TH2

Forward the packet to the next hop (Normal AODV Flow) and exit.

Else:
Initiate alternate path discovery. Neighbours try to find alternate path and update routing table to bypass the current node. Forward the current packet and exit.

\section{SIMULATION MODEL}

The proposed OPS-AODV protocol is implemented in NS2 (NS2.35) and compared with traditional AODV. Energy model is used in NS2 to initialize transmission range $(250 \mathrm{~m})$, initial power (60J) etc. Table1 shows the parameters setting for the simulation setup. The channel bandwidth is set to $2 \mathrm{Mbps}$. For radio propagation, two-ray ground reflection model is used. The traffic type is CBR with packet size 512 bytes. Table 1 shows the simulation parameters.

Table 1. Simulation Parameters

\begin{tabular}{|c|c|}
\hline Type & Values \\
\hline Channel & Channel/Wireless Channel \\
\hline Radio Propagation Model & Propagation/Tworayground \\
\hline Network Interface & Physical/Wirlessphy \\
\hline MAC & MAC/802_11 \\
\hline Interface Queue & Queue/DropTail/PriQueue \\
\hline Antenna & Antenna/Omniantenna \\
\hline Link Layer & LL \\
\hline Routing Protocol & AODV, OPS-AODV \\
\hline Interface Queue Length & 50 \\
\hline Simulation Time & $100 \mathrm{~s}$ \\
\hline
\end{tabular}

Figure 2 shows the typical scenario used with alternate path selected during congestion situation.

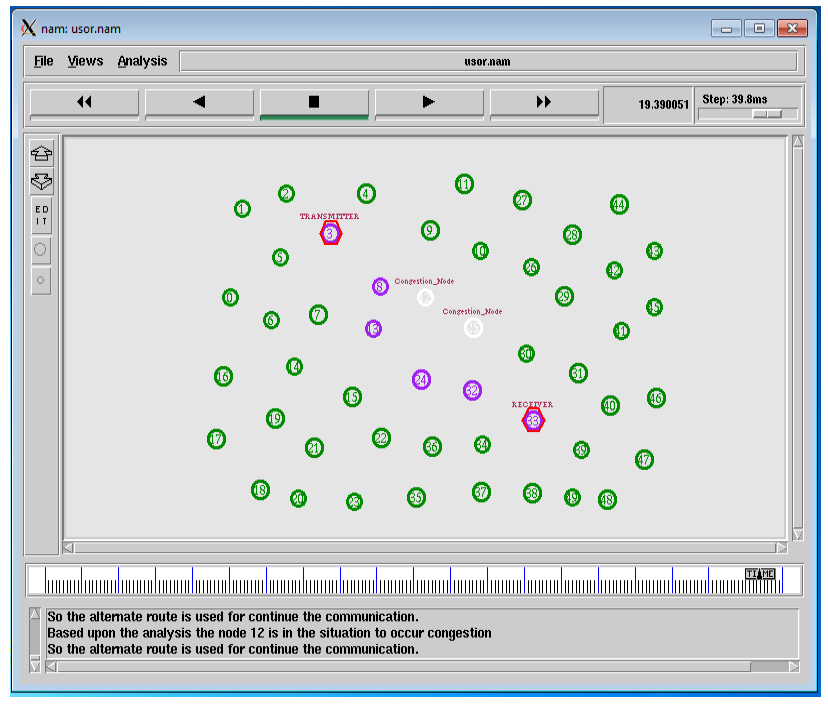

Fig 2: Simulation Scenario

\section{RESULTS}

Two different scenarios are used for experiments:

A. Different number of connection (form 10 to 40).

B. Different node mobility (from 0 to $30 \mathrm{~m} / \mathrm{sec}$ ). 


\section{A. Different number of connection (form 10 to 40):}

Here 50 nodes are used which are randomly scattered in a region of $1000 \mathrm{~m} \mathrm{X} 1000 \mathrm{~m}$. The load on the network is increased in terms of number of connections from 10 to 40 , with mobility $10 \mathrm{~m} / \mathrm{s}$. The cbrgen.tcl and setdest utility is used for traffic and mobility model generation. The performance of the proposed protocol is evaluated and compared with the traditional AODV.

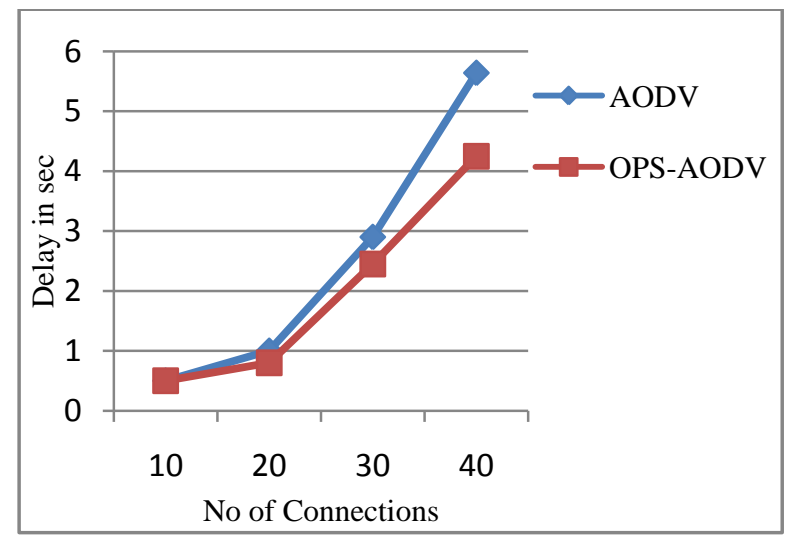

Fig 3: E2E delay Vs No. of Connections

As Figure 3 indicates, at low load condition the delay for both the protocol is same. When the load on the network increases the delay also increases. OPS-AODV incurs less delay compare to traditional AODV at high load condition. This is because of congestion handling mechanism in the OPSAODV which find alternate path in congestion situations.

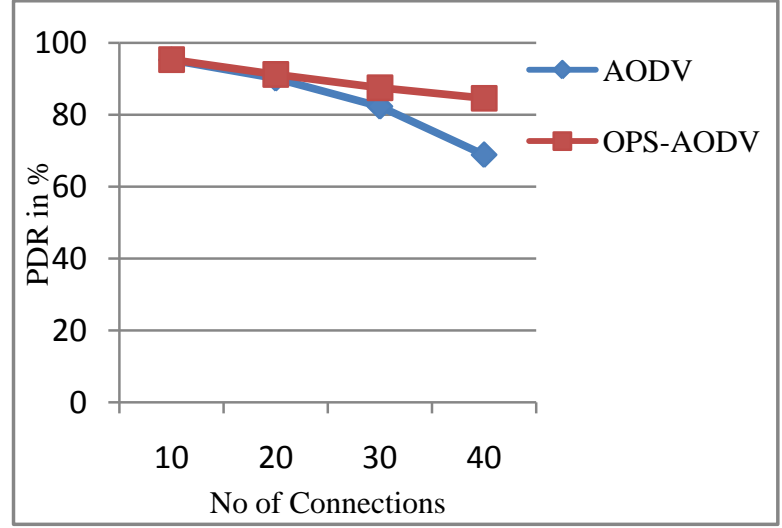

Fig 4: PDR Vs No. of Connections

Figure 4 shows the Packet Delivery ratio and number of connections. The initial PDR for both the protocol is same which decreases as the load increases. The performance of OPS-AODV is better than AODV protocol when the network is tested against higher load.

Figure 5 shows the reduction in power consumed by OPSAODV in high load condition compare to AODV. The reason behind this reduction is that when load increases, the packet loss increases due to congestion, more energy loss is incurred by AODV. OPS-AODV finds alternate route for congested nodes and reduce packet loss. Also OPS-AODV only selects high energy nodes for packet forwarding.

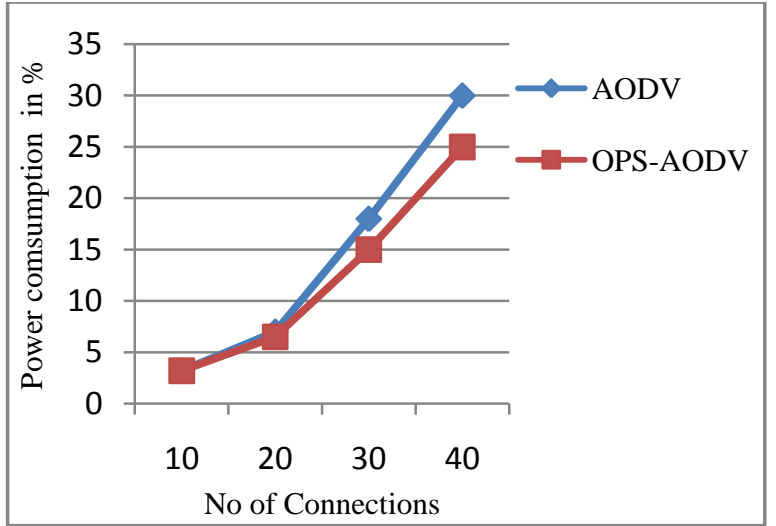

Fig 5: Power consumption Vs No. of Connections

B. Different node mobility ( 0 to $30 \mathrm{~m} / \mathrm{sec}$ )

Here 50 nodes are used which are randomly scattered in a region of $1000 \mathrm{~m} \times 1000 \mathrm{~m}$. The load on the network is kept constant, 10 connections. The mobility of the nodes is increased from 0 to $30 \mathrm{~m} / \mathrm{s}$ in step size of $10 \mathrm{~m} / \mathrm{s}$. The cbrgen.tcl and setdest utility is used for traffic and mobility model generation. The performance of the proposed algorithm is evaluated and compared with the traditional AODV.

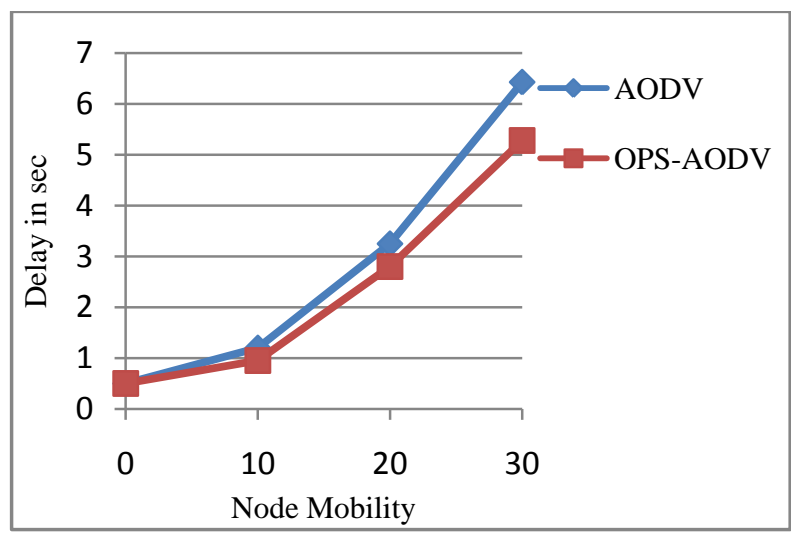

Fig 6: E2E Delay Vs Node Mobility

As Figure 6 indicates, at low mobility condition the delay experienced by both protocols is same. When the network instability increases with higher node velocity the delay also increases. OPS-AODV performs better in this case because of channel monitoring with RNSP mechanism which finds alternate path in unstable channel conditions before the link breaks.

Figure 7 shows the packet delivery ration and the node mobility. Initial PDR is same for both the protocols because the mobility is very low and low mobility do not cause path breaks. As the mobility increases the path breaks occurs due to high mobility and the packet delivery ratio starts decreasing. The results indicate that the performance of proposed OPS-AODV is better than AODV protocol as the node mobility increases, because of link monitoring. 


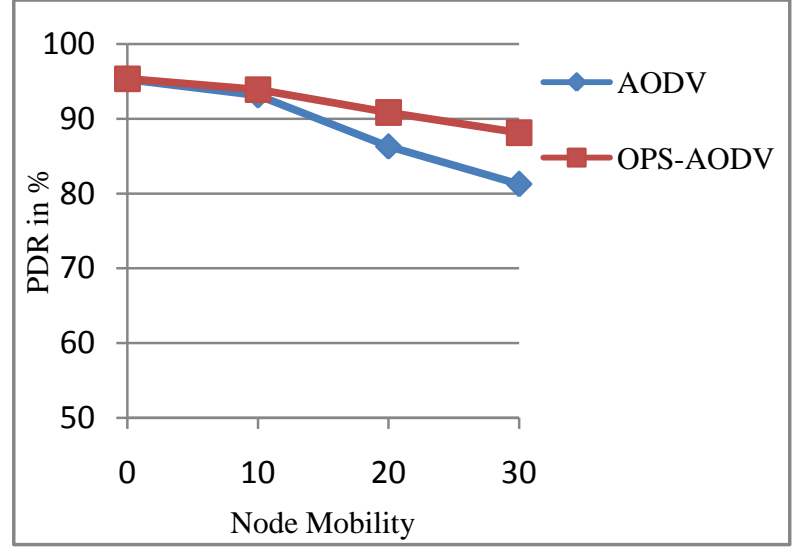

Fig 7: PDR Vs Node Mobility

Figure 8 shows the power consumption of the nodes as the node mobility changes. Both the protocol performs same under initial low mobility condition. When the node mobility increases more path breaks occurs due to mobility, which increases energy consumption of the nodes. Results indicate that OPS-AODV performs better than traditional AODV as the mobility of the nodes increases. The OPS-AODV selects alternate path before the path breaks which reduces control overhead and also high power nodes are selected in routing which reduces average energy consumption and increase life time of the network.

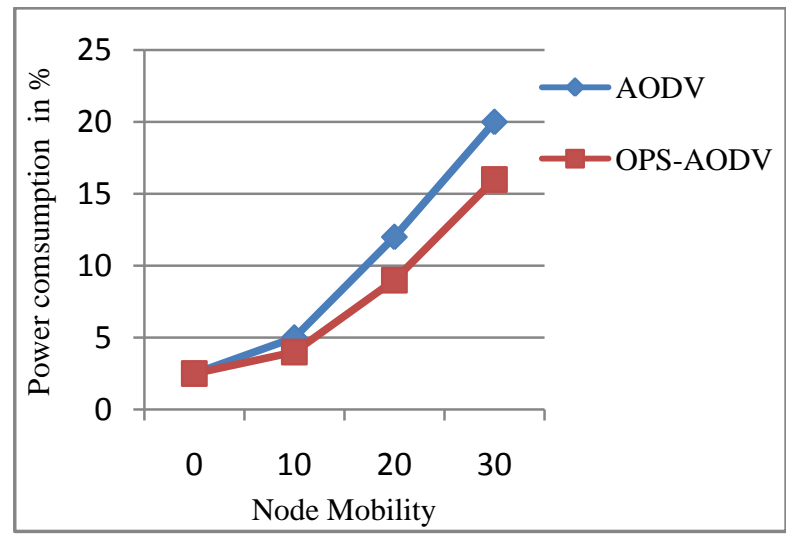

Fig 8: Power consumption Vs Node Mobility

\section{CONCLUSION AND FUTURE WORK}

The paper presents a new cross layer based optimal path selection protocol which considers link status, energy status and congestion status of the nodes for selecting an optimal path between source and destination. The proposed OPSAODV is implemented in NS2 simulator and compared with traditional AODV protocol. The combined energy, congestion and link metric leads to achieve higher packet delivery ratio, lower delay and energy consumption, as indicated in results. Thus proposed OPS-AODV protocol improves the network QoS parameters, reduces energy consumption and increases network life time. The sleep mode in low power nodes can be added as a future work to further save node energy which can be used for only high priority traffic. Similarly broadcast control technique can be incorporated in proposed protocol to further reduce congestion in the network.

\section{REFERENCES}

[1] S. R. Murthy and B. S. Manoj. Ad hoc wireless networks architectures and protocols. Pearson Education, 2007.
[2] D. A. Tran and H. Raghavendra. "Congestion adaptive routing in mobile ad hoc networks". IEEE Trans Parallel Distributed Systems, Vol. 17, No. 11, pp. 16-28, 2006.

[3] S. Lindsey, K. Sivalingam, and C. S. Raghavendra. Power Optimization in Routing Protocols for Wireless and Mobile Networks. Handbook of Wireless Networks and Mobile Computing, I. Stojmenovic, Ed.,Wiley, 2001.

[4] C. Perkins and P. Bhagwat. "Highly dynamic destination-sequenced distance-vector routing for mobile computers". In ACM SIGCOMM'94 Conf. on Comm. Architectures, Protocols and App., pp. 234-244, 1994.

[5] C. E. Perkins and E. M. Royer. "Ad-hoc On-Demand Distance Vector Routing". Proceedings of the Second IEEE Workshop on Mobile Computer Systems and Applications, pp. 90, Feb. 25-26, 1999,

[6] D.B. Johnson, D. A. Maltz and J. Broch. DSR: the dynamic source routing protocol for multihop wireless ad hoc networks. Ad hoc networking, Addison-Wesley Longman Publishing Co., Inc., Boston, MA. 2001.

[7] N. Beijar. Zone Routing Protocol (ZRP). Networking Laboratory, Helsinki University of Technology, Finland. Licentiate course on Telecomm. Technology, 2002.

[8] V. Srivastava and M. Motani. "Cross layer design: a survey and the road ahead". IEEE Comm. Mag., Vol. 43. No. 12, pp. 112-119, 2005.

[9] M. Conti, G. Maselli and G. Turi. "Cross-layering in mobile ad-hoc network design". IEEE Comp. Soc., pp. 48-51, Feb. 2004.

[10] The ns Manual, [Online]. Available: www.isi.edu/nsnam/ns/ns-documentation.html.

[11] V. Rishiwal, A. Kush and S. Verma. "Stable and Energy Efficient Routing for Mobile Ad hoc Networks". IEEE Fifth International Conf. on Information Technology: New Generations, pp. 1028-1033, 2008.

[12] S. Singh, M. Woo and C. S. Raghavendra. "Power-aware routing in mobile ad hoc networks". In Proc. of 4th Annual ACM/IEEE International Conference on Mobile Computing and Networking, pp. 181-190, 1998.

[13] P.K.Suri, M.K.Soni and P. Tomar. "QoS enabled power aware routing protocol (QEPAR)". International Journal of Engineering Science and Technology, Vol. 2, No. 9 , pp. 4880-4885, 2010.

[14] T. S. Kumaran and V. Sankaranarayanan. "Early congestion detection and adaptive routing in MANET". Egyptian Informatics Journal, Elsevier, Vol. 12, No. 3, pp. 165-175, Nov. 2011

[15] A. Nedumaran and V. Jeyalakshmi. "CAERP: A Congestion and Energy Aware Routing Protocol for Mobile Ad Hoc Network". Indian Journal of Science And Technology, Vol. 8, No. 35, 2015.

[16] S. P Terdal, V. D. Mytri and A. Damodaram. "A Load Aware Routing Mechanism for Improving Energy Efficiency in Mobile Ad Hoc Networks". International Journal of Computer Applications, Vol. 10, No. 3, pp. 611, Nov. 2010. 